\title{
Search for New Particles or Gauge Bosons Decaying into Dileptons/Dijets at the Tevatron
}

\author{
Mario Paolo Giordani \\ (for the CDF and DØ collaborations) \\ University of California, Davis - One Shields Avenue, Davis, CA 95616 (USA) - e-mail: giordani@fnal.gov
}

Received: October 14, 2003

\begin{abstract}
The existence of new particles decaying in a jet or lepton pair is probed with the Run II data collected by the Tevatron $\mathrm{p} \overline{\mathrm{p}}$ collider at $\sqrt{s}=1.96 \mathrm{TeV}$. Searches performed on both jet and lepton data collected by the CDF and D $\varnothing$ detectors do not show signs of any new resonance within the considered mass range. The sensitivity achieved by these searches leads to $95 \%$ C.L. limits on the production cross-section times branching ratio for axigluons, flavour universal colorons, excited quarks, colour octet techni- $\rho, \mathrm{E}_{6}$ diquarks, new gauge bosons and Randall-Sundrum gravitons. Excluded mass regions for these models are also computed.
\end{abstract}

PACS. 13.85.Rm, 14.80.-j, 14.70.Pw, 12.60.-i Limits; Other gauge bosons; Other particles; Models

\section{Introduction}

Many extensions of the Standard Model (SM) or alternative models predict the existence of new massive states; due also to the magnitude of their production cross-sections (in some cases expected to be particularly large), the decay rate of these new particles in parton or lepton pairs grants enough sensitivity to constrain their mass and, ultimately, the parameter space of the models predicting their existence. The increased center of mass energy available for the $\mathrm{p} \overline{\mathrm{p}}$ collisions at the Run II of the Tevatron makes these kind of exotic searches particularly interesting even with limited statistics.

\section{Search for New Particles Decaying in Dijets}

Many new particles are predicted to have a larger branching fraction into two partons (quarks and gluons) than into modes containing a lepton or an electroweak gauge boson; nevertheless, frequently the dijet channel is considered to be affected by the large QCD background and the poor mass resolution and therefore not further investigated. However, high statistics in the dijet sample allow exclusions or resonances to the level of a small fraction of the inclusive dijet cross-section. Besides, the limited dijet mass resolution can be turned into an advantage which allows the search for different phenomena by means of a single measured distribution: new particles with a natural width significantly smaller than the measured dijet mass resolution share the same line shape. This one line shape (ultimately dominated by the detector dijet mass resolu- tion) is eventually used for setting an upper limit on crosssection times branching ratio; subsequently, this limit is compared to the predictions of different models as a function of mass in order to obtain excluded mass ranges for new particles having a narrow intrinsic width. CDF, for which the dijet mass resolution is $\sim 10 \%$, uses this procedure to repeat the search for sufficiently narrow resonances performed in Run I[1] at a higher center of mass energy. Specific searches have been conducted for axigluons (A), colorons (C), excited quarks $\left(\mathrm{q}^{*}\right)$, colour octet techni- $\rho$ $\left(\rho_{\mathrm{T}}\right)$, diquarks $(\mathrm{D})$, new gauge bosons $\left(\mathrm{V}^{\prime}=\mathrm{W}^{\prime}, \mathrm{Z}^{\prime}\right)$ and Randall-Sundrum (RS) gravitons (G), as listed in Table 1.

\subsection{Procedure and Results}

The search is based on the $75 \mathrm{pb}^{-1}$ of inclusive jet data collected by CDF in Run II up to winter 2003. Jets are reconstructed with a cone algorithm (semi-aperture $R=0.7$ ) and their energy-momentum corrected to account

\begin{tabular}{lcc}
\hline Model & Particle & $\Gamma / 2$ \\
\hline chiral colour [2] & $\mathrm{A} \rightarrow \mathrm{q} \overline{\mathrm{q}}$ & $0.05 \cdot M_{\mathrm{A}}$ \\
extended technicolor & $\mathrm{C} \rightarrow \mathrm{qg}$ & $0.05 \cdot M_{\mathrm{C}}$ \\
composite fermions [3] & $\mathrm{q}^{*} \rightarrow \mathrm{qg}$ & $0.02 \cdot M_{\mathrm{q}^{*}}$ \\
technicolor [4] & $\rho_{\mathrm{T}} \rightarrow \mathrm{g} \rightarrow \mathrm{q} \overline{\mathrm{q}}, \mathrm{gg}$ & $0.01 \cdot M_{\rho_{\mathrm{T}}}$ \\
superstring-inspired $\mathrm{E}_{6}[5]$ & $\mathrm{D} \rightarrow \mathrm{u} \overline{\mathrm{d}}$ & $0.004 \cdot M_{\mathrm{D}}$ \\
various [6] & $\mathrm{V}^{\prime} \rightarrow \mathrm{q} \overline{\mathrm{q}}\left({ }^{\prime}\right)$ & $0.014 \cdot M_{\mathrm{V}^{\prime}}$ \\
Randall-Sundrum [7] & $\mathrm{G} \rightarrow \mathrm{q} \overline{\mathrm{q}}, \mathrm{g} \overline{\mathrm{g}}$ & $0.06 \cdot M_{\mathrm{G}}$ \\
\hline
\end{tabular}

Table 1. Narrow resonances into dijets investigated by CDF. 
Search for New Particles Decaying to Dijets

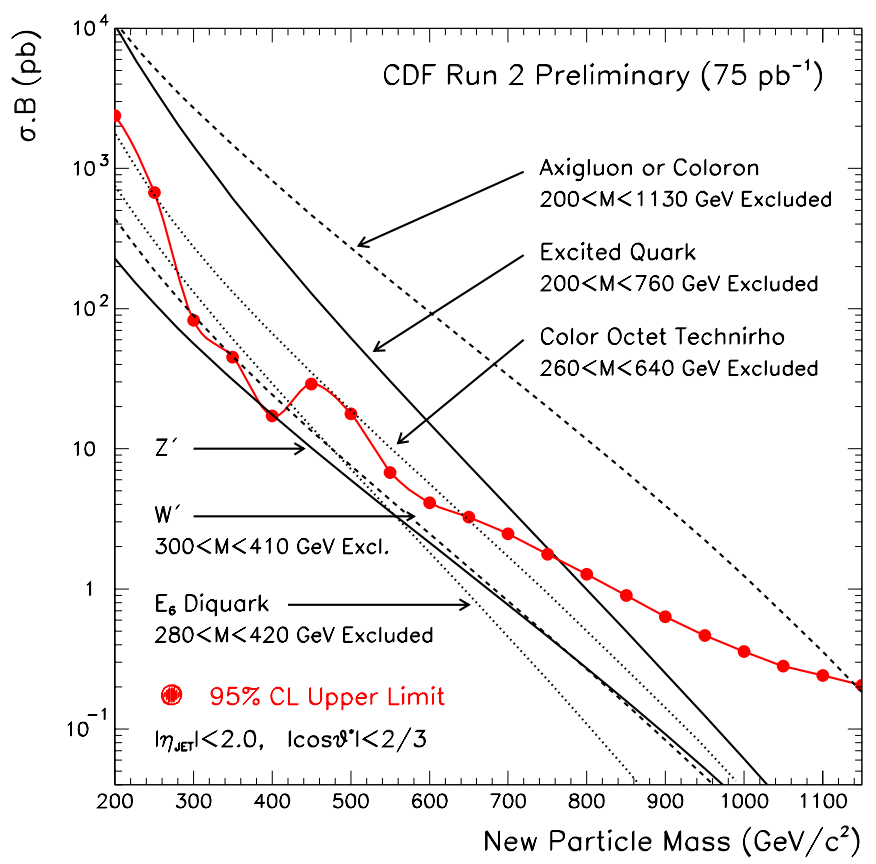

Fig. 1. CDF searches for new particles decaying into dijet: measured $95 \%$ C.L. upper limit on cross-section times branching ratio compared with predictions from various models.

for calorimeter non-linearities, energy losses in uninstrumented regions and outside the cone, or energy contributions from the underlying event. After cosmic ray clean-up, only events having the two leading jets detected in the central region of the detector $\left(\left|\eta_{j e t}\right|<2\right)$ are considered. The dominant background contribution (t-channel QCD) is reduced by applying the angular requirement $\left|\cos \theta^{*}\right| \equiv \tanh \left(\eta_{1}-\eta_{2}\right) / 2<2 / 3$.

The dijet mass distribution is then fit with the sum of a smoothly decreasing function (assumed to describe the QCD background) and of the line shape of a resonance. A signal of excited quark, generated with PYTHIA and processed through the CDF detector simulation, is used to sculpt the actual resonance shape; this is found to be well represented by a Gaussian core with a $\sim 10 \%$ resolution

\begin{tabular}{lc}
\hline New particle & $95 \%$ C.L. excluded mass $\left[\mathrm{GeV} / c^{2}\right]$ \\
\hline axigluon $^{\dagger}$ & $200<M_{\mathrm{A}}<1130$ \\
coloron & $200<M_{\mathrm{C}}<1130$ \\
exited quark & $200<M_{\mathrm{q}^{*}}<760$ \\
techni- $\rho$ & $260<M_{\rho_{\mathrm{T}}}<640$ \\
$\mathrm{E}_{6}$ diquark & $280<M_{\mathrm{D}}<420$ \\
gauge bosons & $300<M_{\mathrm{V}^{\prime}}<410$ \\
$\mathrm{RS}$ graviton & \\
& $220<M_{\mathrm{G}}<840$ \\
\hline$\dagger$ & previous CDF measurement $[8]: 120 \mathrm{GeV} / c^{2}<M_{\mathrm{A}}$ \\
${ }^{\dagger}$ for $k / M_{P L}=0.3$
\end{tabular}

Table 2. Search for new particles into dijets: CDF results. and a radiative tail toward low masses, as expected from QCD radiation.

The results of the fitting procedures have been translated into measured $95 \%$ C.L. upper limits on cross-section times branching ratio and subsequently compared with the theoretical expectations of the different models listed in Table 1. The results are shown in Figure 1 and summarized in Table 2.

\section{Search for New Particles Decaying in Dileptons}

New neutral gauge bosons $\left(\mathrm{Z}^{\prime}\right)$ are expected by many extensions of the SM[6]; these models usually specify the strength of the coupling of such bosons to fermions without making any prediction upon their mass. Although not supported by any theoretical model, a $\mathrm{Z}^{\prime}$ with SM couplings represents a convenient standard reference, since all specific $\mathrm{Z}^{\prime}$ models are expected to have smaller crosssections. A very clean signature of the production of a massive vector boson at hadron colliders is through its decay into a lepton pair $(\ell=\mathrm{e}, \mu)$; this has been exploited in Run I by both CDF [9] and DØ [10]. The same signature is shared by the leptonic decays of RS gravitons [7].

Hence, the search for a high-mass bump in the dilepton spectrum allows to constrain the cross-section times branching ratio for both neutral gauge bosons and RS gravitons, or, equivalently, to provide limits on their masses.

\subsection{Procedure and Results}

CDF searches for new particles decaying in dileptons in inclusive high- $p_{\mathrm{T}}$ electron and muon samples, each accounting for an integrated luminosity of $72 \mathrm{pb}^{-1}$; the search performed by $\mathrm{D} \varnothing$ is based on $50 \mathrm{pb}^{-1}$ of inclusive high- $p_{\mathrm{T}}$ electron data.

Dilepton events $\left(\mathrm{e}^{+} \mathrm{e}^{-}\right.$or $\left.\mu^{+} \mu^{-}\right)$are filtered by means of standard lepton identification selections. For electrons, these include a lower limit on the electromagnetic fraction of the cluster associated to the electron candidate, isolation criteria and cluster shower profile compatible with the hypothesis of being an electron; CDF makes additional use of its tracking capabilities by requiring an $E / P$ cut. Muons are identified by their minimum ionizing nature: their standard selection relies on the quality of the match between a trajectory reconstructed by the inner tracking system of the detector and a track segment detected in the muon systems located outside the calorimeters.

After cross-checking the analyses against the known $\mathrm{Z}^{0} \rightarrow \ell^{+} \ell^{-}$signal, both CDF and DØ high-mass dilepton spectra are compared to Drell-Yan and other SM process expectations and no excess is observed. 95\% C.L. limits are calculated in the assumption of processes dominated by Poisson fluctuations. CDF results are summarized in Figures 2 and 4; DØ excludes (at $95 \%$ C.L.) a $\mathrm{Z}^{\prime}$ decaying into dielectrons for $M_{\mathrm{Z}^{\prime}}<620 \mathrm{GeV} / c^{2}$, as shown in Figure 3 . 

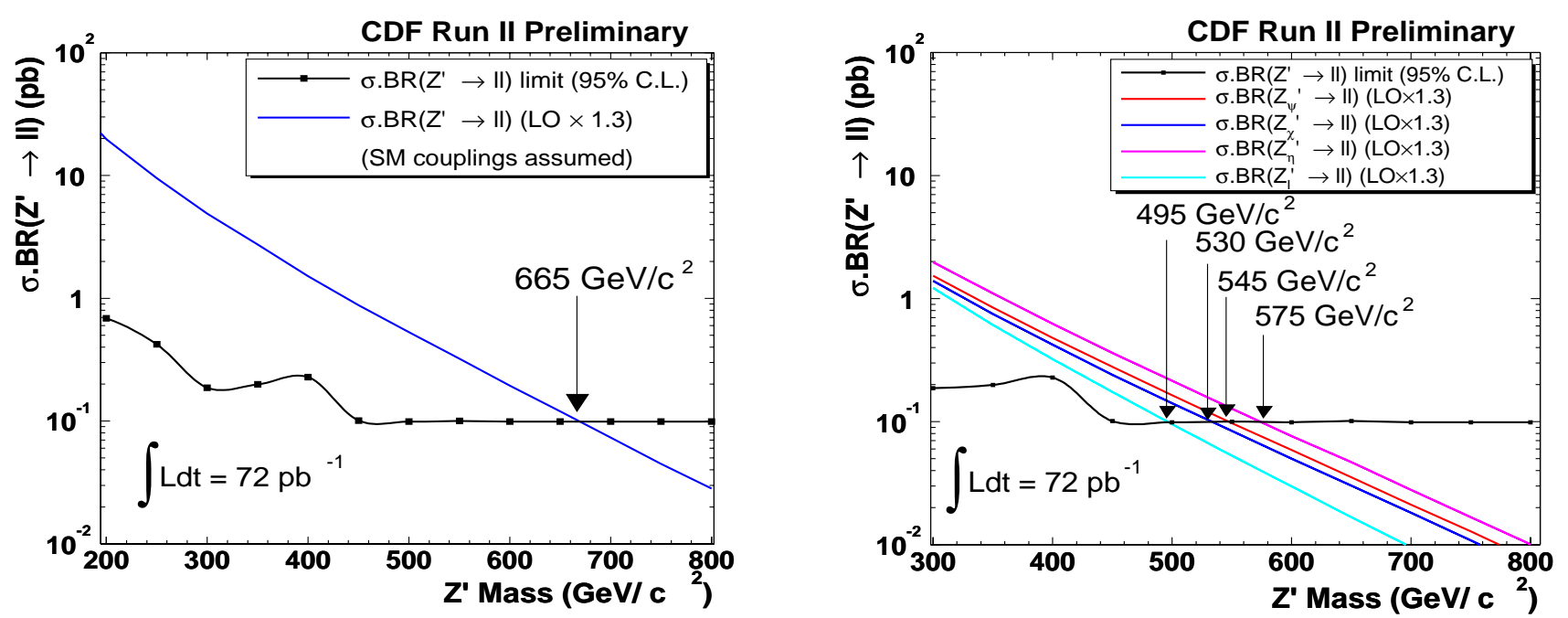

Fig. 2. Searches for new particles decaying into dileptons (CDF): measured 95\% C.L. upper limit on cross-section times branching ratio compared with theoretical predictions from a SM-like and an $\mathrm{E}_{6}$ model $\mathrm{Z}^{\prime}$ (left and right respectively).

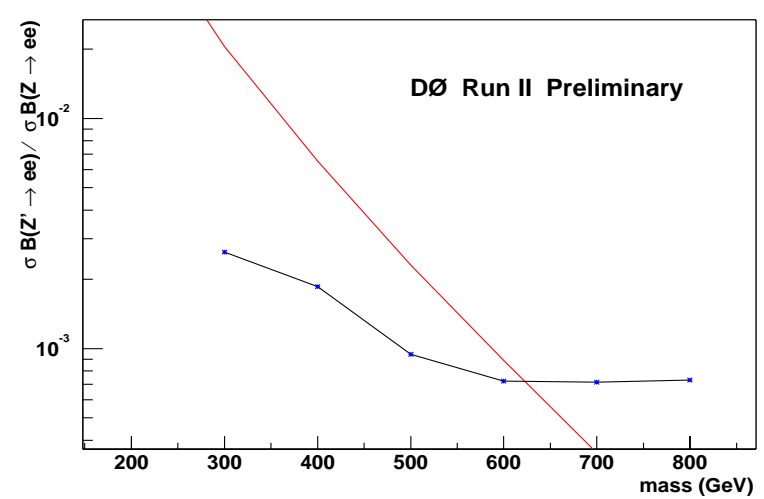

Fig. 3. Searches of new gauge bosons decaying into dielectrons $(\mathrm{D} \varnothing)$ : measured $95 \%$ C.L. upper limit on $(\sigma \cdot B)_{\mathrm{Z}^{\prime}} /(\sigma \cdot B)_{\mathrm{Z}^{0}}$ compared with the theoretical prediction for a SM-like $\mathrm{Z}^{\prime}$.

\section{Conclusions}

CDF and DØ have performed searches for massive particles decaying into jet or lepton pairs on the first Run II data; no evidence has been observed. Despite the lower statistics, these searches have already matched - and in some cases, improved on - the Run I sensitivity.

\section{References}

1. F. Abe et al., Phys. Rev. Lett. 74, (1995) 3538.

2. P. H. Frampton and S. L. Glashow, Phys. Lett. B 190, (1987) 157.

3. U. Baur, I. Hinchliffe and D. Zeppenfeld, Int. J. Mod. Phys. A2, (1987) 1285; U. Baur, M. Spira and P. M. Zerwas, Phys. Rev. D42, (1990) 815.

4. K. Lane and M. V. Ramana, Phys. Rev. D44, (1991) 2678;

E. Eichten and K. Lane, Phys. Lett. B327, (1994) 129.

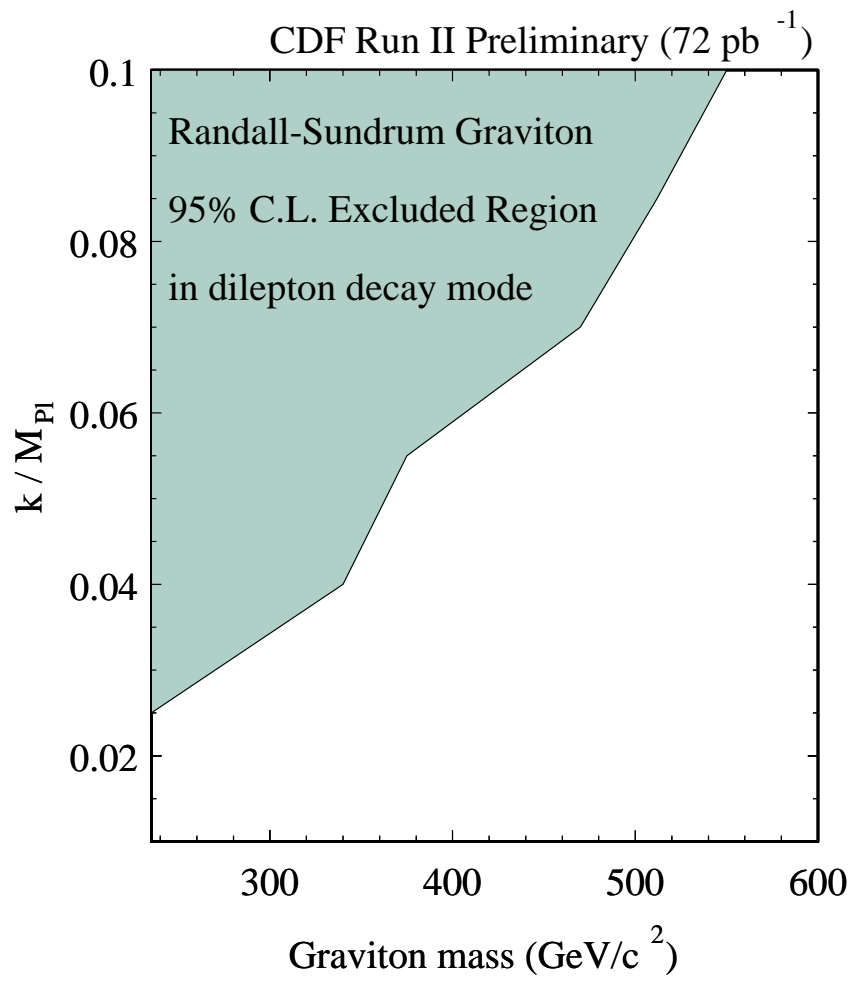

Fig. 4. CDF excluded mass for RS gravitons into dileptons.

5. G. Katsilieris, O. Korakiantis and S. D. P. Vlassopulos, Phys. Lett. B288, (1992) 221; T. G. Rizzo, Phys. Rev. D48, (1993) 4470 and references therein.

6. G. G. Ross, Grand Unified Theories (Cambridge University Press, Cambridge, England, 1987) and references therein.

7. L. Randall and R. Sundrum, Phys. Rev. Lett. 83, (1999) 3370 .

8. F. Abe et al., Phys. Rev. D41, (1990) 1722.

9. F. Abe et al., Phys. Rev. Lett. 79, (1997) 2192.

10. S. Abachi et al., Phys. Lett. B385, (1996) 471. 